\title{
Hyperspectral Estimation of Leaf Area Index of Winter Wheat Based on
} Akaike's Information Criterion

\author{
Haikuan Feng ${ }^{a}$, Fuqin Yang ${ }^{a b c(\bowtie)}$, Guijun Yang ${ }^{a}$, Haojie Pei ${ }^{a b}$ \\ ${ }^{a}$ Beijing Research Center for Information Technology In Agriculture, Beijing, 100097, China e-mail: \\ yangfuqin0202@163.com \\ ${ }^{\mathrm{b}}$ College of Geoscience and Surveying Engineering, China University of Mining \& Technology, Beijing 10083, \\ China \\ ${ }^{c}$ College of Civil Engineering, Henan Institute of Engineering, Zhengzhou ,451191, China
}

\begin{abstract}
Leaf Area Index (LAI) is an important parameter for assessing the crop growth and winter wheat yield prediction. The objectives of this study were (1) to establish and verify a model for the LAI of winter wheat, where the regression models, extended the Grey Relational Analysis(GRA), Akaike's Information Criterion(AIC), Least Squares Support Vector Machine (LSSVM) and (ii) to compare the performance of proposed models GRA-LSSVM-AIC. Spectral reflectance of leaves and concurrent LAI parameters of samples were acquired in Tongzhou and Shunyi districts, Beijing city, China, during 2008/2009 and 2009/2010 winter wheat growth seasons. In the combined model, GRA was used to analyse the correlation between vegetation index and LAI, LSSVM was used to conduct regression analysis according to the GRA for different vegetation index order of the number of independent variables, AIC was used to select the optimal models in LSSVM models. Our results indicated that GRA-LSSVM-AIC optimal models came out robust LAI evaluation (R2 $=0.81$ and 0.80 , RMSE $=0.765$ and 0.733 , i ndividually). The GRA-LSSVM-AIC had higher applicability between different years and achieved prediction of LAI estimation of winter wheat between regional and annual levels, and had a wide range of potential applications.
\end{abstract}

Key words: leaf area index, Akaike's Information Criterion, grey relational analysis, least squares support vector machine

\section{Introduction}

Leaf area index( LAI) means the total region of the plant leaves per unit account for the multiples of the total land area, it is an important indicator for measuring the plant's growing conditions and production forecast, as well as the center hub of reflecting the material production and remote sensing estimation[1, 2].Traditional LAI determination method is depend on direct ground measure, however, for its time-consuming, and disruptive, it is difficult to achieve large area and longtime monitoring. The development of modern remote sensing technology supplies an chance for large area of LAI determination, and it has the advantage of fast determination, timely, low consumption, non-invasive and others. Therefore, it provides a speculative knowledge for monitoring the growth of large area crops, reporting the latest agricultural condition and estimating yield. At present, the LAI inversion methods mainly are empirical statistical model and physical model, and physical model parameter has clear physical meaning, but it requires inputting more parameters and more complex. Though empirical statistical model lacks of strong mechanism, it has the advantage of simple form and convenient calculation, so a lot of domestic and foreign scholars from different perspectives carried out extensive research on the issue of LAI (3-6].Xie Qiaoyun and others[7] have used PCA method to reduce the dimensionality of hyperspectral data and LSSVM model to have LAI inversion of winter wheat under different conditions. It turned out that LS-SVM model has higher estimate ability and universality for winter wheat LAI under different conditions. Lin Hui et al.[8] have used the selected optimum index factor based on LS - SVM algorithm to establish inverse model, and then mapped the remote sensing images, and the result showed that the LS - SVM can be regard as the optimization algorithm for modeling. Cai Qingkong and other researchers [10] have combined the method of PCA and LS- SVM, using multi-band information to establish hyperspectral estimation model of LAI, then compare it with the model obtained by the combination of vegetation index and LS - SVM model, it turned out that the LS-SVM model with the PCA parameter ranks the highest precision model. Jin Xiuliang and others[11,12] have attempted to integrate the method of GRA, SRM and PLS to estimate leaf water content, the result showed that this integrated method can raise the estimate accuracy of winter wheat's leaf water content and more reliable. Xia Tian and others [13] have adopted the regression analysis and BP neural network method to build winter wheat LAI inversion model, and evaluated the inversion precision of the two methods. The result showed that the BP neural network method has greatly improved the estimate accuracy than the regression analysis. Lee et al. [14] have adopted different methods to estimate the LAI of four different plants based on the use of high spectral and multi-spectral data, and the result displayed that the red edge and near infrared region spectrum estimate LAI has higher accuracy than the near infrared spectral range. Jin Shunfa [15] and others have taken the AIC into the polynomial model of crop yield, and the result showed that the AIC criterion can use less parameters to describe the model, and the AIC criterion which is considered to be the best model.

Currently, there are many studies about the estimation of leaf area index, but most studies do not take into account that whether the established model between vegetation index and LAI is the best model. This essay first introduces the gray correlation analysis[12-16] to select the vegetation index, chooses the number of different vegetation indices as input factors according to the relational value, establishes different LAI estimation models based on the LSSVM algorithm and GRA array according to the increasing number of independent variable, and then screens the optimal LAI estimation model of winter wheat according to the ACI. This research offers a new method for the Hyperspectral remote sensing evaluation of the LAI of winter wheat. 


\section{Material and Methods}

\subsection{Brief Introduction of Research Area}

The winter wheat experiments were implemented in 2008/2009 and 2009/2010 in Beijing Shunyi and Tongzhou. Shunyi is located in the northeast of Beijing, located at latitude $40^{\circ} 00^{\prime}-40^{\circ} 18^{\prime}$ ', longitude $116^{\circ} 28^{\prime}$ $116^{\circ} 58^{\prime}$, with an total area of $1,019.89$ square kilometers. Tongzhou is located in southeast of Beijing, located at latitude $39^{\circ} 36^{\prime}-40^{\circ} 02$ ', longitude $116^{\circ} 32^{\prime}-116^{\circ} 56^{\prime}$, with 906 square areas kilometers. During the experiment, two parallel winter wheat fields were chosen as experiment plots, and respectively conducted the canopy spectrometric and plants sampling in the critical growth period of winter wheat.

\subsection{Canopy Spectrometry}

The research introduces ASD FieldSpec FR2500 spectrometer to sample winter wheat from 10am to 2pm Beijing time in Tongzhou and Shunyi districts when the weather was sunny and windless. During the observation, the probe is vertically downward, and at the height of 1.3 meters from the ground, with $25^{\circ}$ field angle. Each sample point was collected 10 times and the average value was regarded as the spectral reflectance of the sample, and reference board calibration was carried out immediately before and after the measurement. The wavelength range of American ASD FieldSpec FR2500 field spectrometer is from $350 \mathrm{~nm}$ to $2500 \mathrm{~nm}$, in which the band range from $350 \mathrm{~nm}$ to $1000 \mathrm{~nm}$ is $1.4 \mathrm{~nm}$, and within the band range of $1000 \sim 2500 \mathrm{~nm}$ is $2 \mathrm{~nm}$. Spectral resampling interval is $1 \mathrm{~nm}$.

\subsection{LAI Determination}

Specific leaf weight method is used for the determination of winter wheat LAI, and the sampling area is 60 $\mathrm{cm} * 60 \mathrm{~cm}$, then the sampling plants were brought to the lab to determine LAI. The LAIcalculation formula is:

$$
\mathrm{LAI}=\frac{\mathrm{W}_{1}+\mathrm{W}_{2}+\mathrm{W}_{3}}{\mathrm{~W}_{1} \times A \times 10000} \times S
$$

$W_{1}$ stands for leaf weight $(\mathrm{g}), \mathrm{S}$ stands for leaf area $\left(\mathrm{cm}^{2}\right), \mathrm{W}_{2}$ refers to the rest leaf weight of 10 stems $(\mathrm{g})$, A stands for the sampling area $\left(\mathrm{m}^{2}\right), W_{3}$ stands for the rest plant leaf weight (g).

\subsection{Method}

\subsubsection{Vegetation Index Selection}

According to the results of previous studies and the significant relationship between vegetation indices and LAI, 14 vegetation indices related to leaf area index were sort out. (table 1)

Table 1 Summary of spectral indices related to LAI

\begin{tabular}{|c|c|c|}
\hline Vegetation Index & Formula & Reference \\
\hline $\begin{array}{l}\text { Normalized difference } \\
\text { vegetation index (NDVI) }\end{array}$ & $\left(\mathrm{R}_{800}-R_{670}\right) /\left(R_{800}+R_{670}\right)$ & {$[18]$} \\
\hline Simple Ratio Index（SR） & $\mathrm{R}_{800} / R_{670}$ & [19] \\
\hline $\begin{array}{c}\text { optimized soil adjusted } \\
\text { vegetation index (OSAVI) }\end{array}$ & $1.16\left(\mathrm{R}_{800}-\mathrm{R}_{670}\right) /\left(R_{800}+R_{670}+0.16\right)$ & {$[20]$} \\
\hline $\begin{array}{l}\text { Transformed chlorophyll } \\
\text { absorption in reflectance index } \\
\text { (TCARI) }\end{array}$ & $3\left[\left(\mathrm{R}_{700}-\mathrm{R}_{670}\right)-0.2\left(\mathrm{R}_{700}-\mathrm{R}_{550}\right)\left(\mathrm{R}_{700} / R_{670}\right)\right]$ & [21] \\
\hline TCARI/OSAVI & TCARI/OSAVI & {$[22]$} \\
\hline $\begin{array}{l}\text { Normalized difference } \\
\text { vegetation index } 705 \\
(\text { NDVI705) }\end{array}$ & $\left(\mathrm{R}_{750}-R_{705}\right) /\left(R_{750}+R_{705}\right)$ & [24] \\
\hline $\begin{array}{l}\text { Modified Red Edge Simple } \\
\text { Ratio Index (mSR705) }\end{array}$ & $\left(\mathrm{R}_{750}-R_{445}\right) /\left(R_{705}-R_{445}\right)$ & [25]] \\
\hline $\begin{array}{l}\text { Modified Red Edge Normalized } \\
\text { Difference Vegetation Index } \\
\text { (mNDVI705) }\end{array}$ & $\left(\mathrm{R}_{750}-R_{705}\right) /\left(R_{750}+R_{705}-2 R_{445}\right)$ & [25] \\
\hline Vogelmann Index（VOG1） & $\mathrm{R}_{740} / R_{720}$ & [26] \\
\hline $\begin{array}{l}\text { Structure Insensitive Pigment } \\
\text { Index (SIPI) }\end{array}$ & $\left(\mathrm{R}_{800}-R_{450}\right) /\left(R_{800}-R_{680}\right)$ & [27] \\
\hline
\end{tabular}




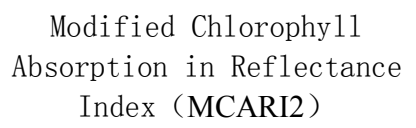

Modified soil adjusted vegetation index (MSAVI2)

Modified Simple Ratio (MSR)

$$
\begin{gathered}
\frac{1.5\left[2.5\left(\mathrm{R}_{800}-\mathrm{R}_{670}\right)-1.3\left(\mathrm{R}_{800}-R_{550}\right)\right]}{\sqrt{\left(2 R_{800}+1\right)^{2}-\left(6 R_{800}-5 \sqrt{R_{670}}-0.5\right.}} \\
\frac{1}{2}\left[2 R_{800}+1-\sqrt{\left(2 R_{800}+1\right)^{2}-8\left(R_{800}-R_{670}\right)}\right] \\
\left(R_{800} / R_{670}-1\right) / \sqrt{R_{800} / R_{670}+1}
\end{gathered}
$$

\subsubsection{Grey Relational Analysis, GRA}

Grey correlation degree is a dimensionless quantity, it is used to identify whether there is a connection between the reference number and comparative sequence, and the specific relation between them. In this study, the concept of grey correlation degree is conducted to calculate the correlation degree between LAI and vegetation index, the higher grey correlation degree, the more closely between vegetation indices and LAI, and vice versa.

\subsubsection{Akaike's Information Criterion (AIC)}

AIC [16] is put forward by H.Akaike during the study of information theory, especially in solving the problem of time series pricing. The AIC formula can be written as:

$\mathrm{AIC}=(-2) \ln$ (maximum likelihood function of the model) +2 (the number of independent parameters of the model)

As for linear regression model, the AIC formula is:

$$
\mathrm{AIC}=\mathrm{n} \ln \left(S_{p}^{2}\right)+2(k+1)
$$

$\mathrm{n}$ is the sample size; $S_{p}^{2}$ stands for residual mean square; $\mathrm{k}$ is the number of variables of the model.

\subsubsection{Least squares support vector machine (LSSVM)}

The basic idea of LSSVM regression is to discovery a best separating hyperplane so that the deviation between the training samples and the optimal hyperplane is minimum, and the linear regression function built in highdimensional feature space is:

$$
f(x)=w \Phi(x)+b
$$

$\Phi(x)$ stands for nonlinear mapping function, and this study selects radial basic function as kernel radial ; $\mathrm{f}(\mathrm{x})$ stands for the predicted value of the regression function.

\subsection{Statistical Analysis}

In this study, determination coefficient $\left(R^{2}\right)$ and RMSE were regarded as indicators to interpret and quantify the relationship between LAI and vegetation index. Generally speaking, the higher $\mathrm{R}^{2}$, the smaller RMSE, and the better ability of the estimate model between the LAI and vegetation index, otherwise, the estimate ability is poor.

\section{Results and Analysis}

\subsection{Correlation between Vegetation Index and LAI}

As can be caught sight of from table 2, there is a significant correlation between vegetation index and LAI, in which TCARI has a noteable relationship with LAI $(\mathrm{p}<0.05)$, and other vegetation indices also have noteable correlation with LAI $(\mathrm{p}<0.01)$. TCARI/OSAVI、SIPI and PRI all have highly significant negative correlation with LAI, and NDVI、SR、OSAVI、NDVI705、MSR705、mNDVI705、VOG1、MCARI2、MSAVI、 MSR have significantly positive correlation with LAI.

Table 2 Correlation between vegetation index and LAI

\begin{tabular}{cccc}
\hline Vegetation index & $\mathrm{r}$ & Vegetation index & $\mathrm{r}$ \\
\hline NDVI & $0.738^{* *}$ & mNDVI705 & $0.734^{* *}$ \\
SR & $0.788^{* *}$ & VOG1 & $0.796^{* *}$ \\
OSAVI & $0.786^{* *}$ & SIPI & $-0.555^{* *}$ \\
TCARI & $0.260^{*}$ & PRI & $-0.793^{* *}$ \\
TCARI/OSAVI & $-0.481^{* *}$ & MCARI2 & $0.815^{* *}$ \\
NDVI705 & $0.777^{* *}$ & MSAVI & $0.813^{* *}$ \\
MSR705 & $0.752^{* *}$ & MSR & $0.811^{* *}$ \\
\hline
\end{tabular}

Note: $* *$ and $*$ represent significant at the 0.01 and 0.05 levels of probability respectively.

\subsection{Grey Correlation between Vegetation Index and LAI}

Table 3 indicates the order of gray correlation degree between vegetation indices and LAI foundation on the analysis of GRA: VOG1 $>$ SIPI $>$ MCARI2 $>$ NDVI $>$ MSR $>$ mNDVI705 $>$ OSAVI $>$ NDVI705 $>$ TCARI / OSAVI $>$ 
TCARI $>$ PRI $>$ MSAVI $>>$ MSR705 $>$ SR. During the gray correlation analysis between vegetation indices and LAI of winter wheat, the correlation degree of VOG1 is 0.921 and ranks first; while SR has the lowest value with the correlation degree 0.612 . and the correlation degree is 0.612 .

Table 3 Grey relational degree and rank of evaluation index for LAI at the whole growth stages

\begin{tabular}{cccccc}
$\begin{array}{c}\text { Evaluation } \\
\text { index }\end{array}$ & $\begin{array}{c}\text { Grey } \\
\text { correlation } \\
(\varepsilon=0.5)\end{array}$ & orders & $\begin{array}{c}\text { Evaluation } \\
\text { index }\end{array}$ & $\begin{array}{c}\text { Grey } \\
\text { correlation } \\
(\varepsilon=0.5)\end{array}$ & orders \\
\hline NDVI & 0.8777 & 4 & mNDVI705 & 0.8715 & 6 \\
SR & 0.6178 & 14 & VOG1 & 0.9211 & 1 \\
OSAVI & 0.8712 & 7 & SIPI & 0.8818 & 2 \\
TCARI & 0.8416 & 10 & PRI & 0.8405 & 11 \\
TCARI/OSAVI & 0.8438 & 9 & MCARI2 & 0.8818 & 3 \\
NDVI705 & 0.8671 & 8 & MSAVI & 0.8405 & 12 \\
MSR705 & 0.8263 & 13 & MSR & 0.8765 & 5 \\
\hline
\end{tabular}

\subsection{The use of AIC to Determine the Optimal Regression Model}

When the independent variables of vegetation index in the equation participated in the LSSVM regression according to their grey correlation degree, and the AIC value has changed in all the regression equation. As can be seen from table 4, when two vegetation index independent variables involved in the LSSVM regression, and the maximum AIC value is 19.055; when five vegetation indices involved in LSSVM regression, and the minimum AIC value is 16.29. The aim of AIC is to select a model that can better interpret the data and contain minimum free parameters, so the model that choosing five vegetation indices involved in the progress of LSSVM regression is regarded as the optimal equation.

Table4 Comparison of LSSVM regression built by different independent variables

\begin{tabular}{cc}
\hline $\begin{array}{c}\text { number of independent } \\
\text { variables }\end{array}$ & AIC \\
\hline 1 & 9.04 \\
2 & 19.06 \\
3 & -0.75 \\
4 & 3.77 \\
5 & -16.29 \\
6 & -1.62 \\
7 & -10.66 \\
8 & 6.97 \\
9 & 13.78 \\
\hline
\end{tabular}

\subsection{Estimation and Validation of LAI}

Through the analysis of section 2.3 and combining the data of whole growth period of winter wheat in 2008/2009 Beijing tongzhou and shunyi districts, the optimal estimate model GRA -LSSVM-AIC LAI is established, in which five vegetation indices are regarded as the independent variables, and the five vegetation indices respectively are SIPI, MCARI2, NDVI, MSR. The estimated results are shown in table 5 and figure 1.As can be seen from table 5 and figure 1 , the value of $\mathrm{R}^{2}$ and RMSE respectively are 0.81 and 0.765 in the GRA-AICLSSVM estimate model, and its modelling accuracy is preferable. In order to verify the precision of the model, the whole winter wheat growth stage in Beijing Tongzhou and Shunyi districts are tested, and the value of $\mathrm{R}^{2}$ and RMSE respectively are 0.80 and 0.733 , and the scatter diagram of predicted value and measured value also have high consistency. It showed that the GRA -LSSVM-AIC model for leaf area estimation is much more reliable.

Table 5 Estimation and validation of LAI

\begin{tabular}{ccccccc}
\hline \multirow{2}{*}{$\begin{array}{c}\text { Modeling } \\
\text { Methods }\end{array}$} & \multicolumn{3}{c}{ Modeling } & \multicolumn{3}{c}{ Validation } \\
\cline { 2 - 6 } & Samples & $\mathrm{R}^{2}$ & RMSE & Samples & $\mathrm{R}^{2}$ & RMSE \\
\hline \multirow{2}{*}{ LSSVM } & 84 & 0.81 & 0.765 & 85 & 0.80 & 0.733 \\
\hline
\end{tabular}





Fig.1 Relationship between predicted LAl and measured LAI using GRA -LSSVM-AIC

(a) modeling and (b) validation

\section{Conclusions}

In order to define the number of the optimal principal components of winter wheat in the model of LSSVM, this essay attempts to use GRA to assess the correlation between vegetation index and winter wheat LAI, and regards LSSVM regression pattern with the minimum AIC value as the best model of the estimation of winter wheat LAI. Namely, the best estimation model of winter wheat LAI is foundation on the integration methods of GRA, LSSVM and AIC. Here comes the following conclusion;

Firstly, the use of GRA to evaluate the correlation sequence between vegetation indices and winter wheat LAI, namely VOG1>SIPI> MCARI2> NDVI> MSR > mNDVI705> OSAVI> NDVI705> TCARI / OSAVI > TCARI $>$ PRI $>$ MSAVI> MSR705> SR. Among them, VOG1 has the highest correlation degree with winter wheat LAI, while SR has the lowest correlation degree.

Then 9 inverse model of winter wheat LAI is established with the adoption of LSSVM algorithm and gradul increase of the number of independent variables according to the GRA order. Then 9 inverse model of winter wheat LAI based on the AIC is optimally filtered, and the winter wheat LAI model consists of 8 independent variables is considered the best model, and the 8 variables are VOG1、SIPI、MCARI2、NDVI、MSR、mNDVI705、

OSAVI、NDVI705.

Third, the modeling value set of $\mathrm{R}^{2}$ and RMSE in the GRA -LSSVM-AIC model respectively are 0.81 and 0.765 , and the value of validation set of $\mathrm{R}^{2}$ and RMSE are 0.80 and 0.733 . The result showed that the GRALSSVM-AIC model used as optimal estimation model for winter wheat LAI not only can be used for the detection of winter wheat LAI of different annual, but also has high modeling accuracy, so it can be regarded as the preferred model for the estimation of winter wheat LAI.

\section{References}

[1] Wang Jihua, Zhao Chunjiang, Huang Wenjiang, et al. Quantitative remote sensing and its application in agriculture[M]. Beijing: Thomson Learning Press, 2008.

[2] Pinter, P.J., Hatfield, J.L., Schepers, J.S., Barnes, E.M., Moran, M.S., Daughtry, C.S., Upchurch, D.R. Remote sensing for crop management. Photogramm. Eng. Remote Sens. 2003, 69, 647-664.

[3] Maki, M., Homma, K. Empirical regression models for estimating multiyear leaf area index of rice from several vegetation indices at the field scale. Remote Sens. 2014, 6, 4764-4779.

[4] Potithep, S., Nagai, S., Nasahara, K.N., Muraoka, H., Suzuki, R. Two separate periods of the LAI-VIs relationships using in situ measurements in a deciduous broadleaf forest. Agric. For. Meteorol. 2013, 169, 148-155.

[5] Inoue, Y., Iwasaki, K. Spectral estimation of radiation absorptance and leaf area index in corn canopies as affected by canopy architecture and growth stage. Jpn. J. Crop Sci. 1991, 60, 578-580.

[6] Li, F., Miao, Y., Hennig, S.D., Gnyp, M.L., Chen, X., Jia, L., Bareth, G. Evaluating hyperspectral vegetation indices for estimating nitrogen concentration of winter wheat at different growth stages. Precis. Agric. 2010, 11, 335-357.

[7] Qiaoyun Xie, Wenjiang Huang, Dong Liang, et al. Comparative Study on Remote Sensing Invertion Methods for Estimating Winter Wheat Leaf Area Index [J]. Spectroscopy and Spectral Analysis.2014, 34(5):489-493.

[8] Lin Hui, Liang Liang, Zhang Lianpeng, et al. Wheat leaf area index inversion with hyperspectral remote sensing based on support vector regression algorithm[J]. Transactions of the Chinese Society of Agricultural Engineering (Transactions of the CSAE), 2013, 29(11): 139-146.

[9] Liang Liang, Yang Minhua, Zhang Lianpeng, et al. Chlorophyll content inversion with hyperspectral technology for wheat canopy based on support vector regression algorithm[J]. Transactions of the Chinese Society of Agricultural Engineering (Transactions of the CSAE), 2012, 28(20): 162-171.

[10] Qingkong Cai, Jinbao Jiang, Liangliang Tao, et al. Estimation of Winter Wheat Leaf Area Index with Joint Principal 
Component Analysis and Least Squares Support Vector Model[J]. Journal of Triticeae Crops.2014, 34(9):1292-1296.

[11] JIN Xiu-liang,XU Xin-gang, WANG Ji-hua, et al. Hyperspectral Estimation of Leaf Water Content for Winter Wheat Based on Grey Relational Analysis[J]. TSpectroscopy and Spectral Analysis, 2012, 32(11): 103-3106.

[12] Xiuliang JIn, Xinggang Xu, Xiaoyu Song, et al. Estimation of Leaf Water Content in Winter Wheat Using Grey Relational Analysis - Partial Least Squares Modeling with Hyperspectral Data [J]. Agronomy Journal, 2013, 105(5): 1085-1392

[13] Xia Tian, Wu Wenbin, Zhou Qingbo, et al. Comparison of two inversion methods for winter wheat leaf area index based on hyperspectral remote sensing[J]. Transactions of the Chinese Society of Agricultural Engineering (Transactions of the CSAE), 2013, 29(3): 139-147. (in Chinese with English abstract)

[14] Kyu-Sung Lee, Warren B.Cohen, Robert E.Kennedy, Thomas K. Maiersperger, Stith T. Gower. Hyperspectral versus multispectral data for estimating leaf area index in four different biomes. Remote Sensing of Environment. 91 (2004)508520.

[15] Jin Shun-fa, Wang Yi-bao, Xu Yu-li, et al. AIC principle and its Application in the Polynomial Models of the Crop Yield[J]. Acta Agriculture Shanghai, 1985, 1(3):73-78.

[16] Zhenhai Li, Xingang Xu, Xiuliang Jin, et al. Remote Sensing Prediction of Winter Wheat Protein Content Based on Nitrogen Translocation and GRA-PLS Method[J]. Scientia Agricultura Sinica, 2014, 47(19):3780-3790.

[17] Akaike.H. Problem of control and information,Proc.2nd Int.Symp.on Information Theory(B.N.Petrov and F. Csaki,eds.),267-281.Akademina kiado,Budapest,1973

[18] Deering D W, Harlan J C. Monitoring the Vernal Advancement and Retrogradation (greenwave effect) of Natural Vegetation. Texas A \& M University, Remote Sensing Center, 1974.

[19] Pearson R L, Miller D L. Remote mapping of standing crop biomass for estimation of the productivity of the shortgrass prairie. Proceedingsof the English International Sysposium on Remote Sensing of Environment, 1972, 2: 1375-1381.

[20] Rondeaux G, Steven M, Baret F. Optimization of soil-adjusted vegetation indices [J]. Remote Sensing environment, 1996, 55(2): $95-107$

[21] Haboudane D, Miller J R, Tremblay N, et al. Integrated narrow-band vegetation indices for prediction of crop chlorophyll content for application to precision agriculture [J]. Remote Sensing of Environment, 2002, 81(2/3): 416 - 426.

[22] Rondeaux G, Steven M, Baret F. Optimization of soil-adjusted vegetation indices [J]. Remote Sensing environment, 1996, 55(2): 95- 107 .

[23] Sims D A, Gamon J A. Relationships between leaf pigment content and spectral reflectance across a wide range of species, leaf structures and developmental stages [J]. Remote Sensing of Environment, 2002, 81(2): 337-354.

[24] Gitelson A, Merzlyak M N. Spectral reflectance changes associated with autumn senescence of aesculus Hippocastanum L. and Acer Platanoides L. leaves. spectral features and relation to chlorophyll estimation[J]. Journal of Plant Physiology, 1994, 143(3): 286-292.

[25] Vogelmann, J E, Rock B N, Moss D M. Red edge spectral measurements from sugar maple leaves [J]. International Journal of Remote Sensing, 1993, 14(8): 1563-1575.

[26] Penuelas J, Baret F, Filella I. Semi-empirical indices to assess carotenoids/chlorophyll a ratio from leaf spectral reflectance. Photosynthetica, 1995, 31(2): 221-230.

[27] Gamon J A, Penuelas J, Field C B. A narrow-waveband spectral index that tracks diurnal changes in photosynthetic efficienc [J]. Remote Sensing of Environment, 1992, 41(1): 35-44.

[28] Haboudane, D., Miller, J.R., Pattey, E., et al., 2004. Hyperspectral vegetation indices and novel algorithms for predicting green LAI of crop canopies: modeling and validation in the context of precision agriculture. Remote Sens. Environ. 90, $337-352$.

[29] Qi J., Chehbouni A., Huete A. R., et al. A Modified Soil Adjusted Vegetation Index. Remote Sensing of Environment, 1994, 48 (2): 119-126

[30] Chen, J. .Evaluation of vegetation indices and modified simple ratio for boreal applications. Can. J. Remote Sens, $1996,22,229-242$ 